dédaigner : avant de commencer les travaux d'une semblable installation, il serait possible d'en fixer à quelques francs près la dépense, - d'où la fixation assez certaine du prix de revient de l'énergie - calcul qui est, comme le savent tous les ingénieurs, d'une exactitude généralement très problématique quand il s'agit de barrages, canaux et usine à établir sur un terrain qui offre des surprises et pour utiliser un débit qui en réserve encore de plus désagréables.

Cette initiative vaut qu'on l'encourage; puissions-nous avorr contribué à porter l'attention sur elle.

E. C.

\section{L'Électricité et la Police correctionnelle}

\section{COUR D'APPEI DE NANCY \\ Audience du 13 juillet 1904.}

Présidence de $M$. DE Luxer.

CONDAMNATION POUR VOL D'ÉLECTRICITÉ.

L'électricité est susceptible d'une propriété privée par l'accumulation qui en est faite, la direction qu'on lui donne, l'usage auquel on l'emploie: force, chaleur ou lumière $\left(^{*}\right)$.

Par suite, commet un vol celui qui, pour faire fonctionner des lampes, se sert du courant transmis par une usine électrique, en l'empêchant de passer par le compteur.

(Viller contre fabius Henrion).

La Gour,

Attendu qu'il résulte, de l'information et des débats, la preuve que, le if mars I 904 , le monteur Michel, attaché à l'usine électrique de Fabius Henrion, à Pagny-sur-Moselle, s'étant rendu chez le prévenu Viller, abonné à l'usine, pour y vérifier son compteur, remarqua que les deux bornes saillantes de l'appareil étaient réunies par deux fils et que, grâce à cette disposition, ajoutée subrepticement, le courant électrique arrivait directement aux lampes sans passer par le compteur, qui se trouvait hors circuit et marquait le chiffre de 1721 .

Attendu que, le 22 mars suivant, le même monteur, se rendant de nouveau chez Viller, constata avec le contremaitre Rougelowski, qui l'assistait, que le compteur était toujours hors circuit et yu'il continuait à indiquer le chiffre de $172 \mathrm{I}$, bien que, dans l'intervalle des deux visites, les lampes eussent été allumées; qu'en ayant fait l'observation à Viller, celui-ci monta vivemement sur son établi et sépara les fils en s'écriant: "Vous pouvez certifier l'avoir vu, moi, je nierai ";

Artendu que, le lendemain, lorsque la gendarmerie procéda à son enquête, le compteur indiquait i 726 , ce qui démontre, d'une part, que la consommation quotidienne de Viller pouvait être évaluée à environ 5 hectowatts;

Attendu que l'attitude de Viller, lors de la visite des employés de Fabius Henrion, son empressement à rétablir la communication, les paroles qu'il a prononcées à ce moment établissent jusquà la dernière évidence que lui seul, quoiqu'il prétende le contraire, est l'auteur de la mise hors circuit du compteur; que le moyen mis en pratique pour y arriver dénote, d'ailleurs, une certaine habileté professionnelle de la part de celui qui y a eu recours; que cette habileté se rencontre précisément chez Viller, qui a aidé le contremaître Rougelowski dans ses installations électriques, et, par suite, acquis des connaissances pratiques qui lui ont servi pour la réalisation de la combinaison fraudu-

(*) Cette théorie n'est pas admise dans tous les pays étrangers. La jurisprudence allemande s'est prononcée en sens contraire. Le Tribunal de l'Empire, par arrêt du 20 octobre 1896 , a admis que dans la définition du vol, le mot choses s'entendait seulement des objets corporéls. Or, d'après les dernières données de la science, l'électricité ne serait pas un fluide, un courant circulant le long-d'un fil, mais une énergie résidant dans le corps électrisé et résultant d'une vibration particulière de ce corps. Devant cette jurisprudence, le législateur allemand a dû, pour ne pas laisser impuni le détournement d'énergie électrique, en faire un délit particulier puni par une loi du 9 avril 1900 . Il existe de même une loi spéciale en Angleterré(loi du 18 août I 882 ). leuse à laquelle il s'est livré dans le but de s'approprier la chose d'autrui ;

Attendu, en effet, que quel que soit l'aspect sous lequel l'électricité doive être envisagée, qu'on la consìdère comme un Huide ou une énergie, il est incentestable qu'elle est susceptible d'une propriété privée par l'accumulation qui en est faite, la direction qu'on lui donne, l'usage auquel on l'emploie, force, chaleur ou lumière; qu'en consommant donc sans la payer, puisque le compteur par lequel elle ne passait plus n'enregistrait pas son débit, une certaine quantité d'électricité, Viller a ainsi fait mainmise, à l'insu de son propriétaire Fabius Henrion, et contre son gré, sur une chose qui ne lui appartenait pas et se l'est appropriée frauduleusement ;

Attendu qu'on objecterait vainement qu'en l'éspèce il ne peut $\mathrm{y}$ avoir appréhension frauduleuse, puisque l'électricité était remise volontairement par Fabius Henrion à Viller; que cette remise, tout d'abord, était subordonnée à la condition que l'électricité passât par le compteur ; qu'il est certain, d'autre part, que Fabius Henrion gardait jusqu'au compteur la propriété exclusive de l'électricité qu'il avait produite et que c'est seulement lorsqu'elle avait franchi l'appareil, après y avoir été enregistrée, qu'elle était remise à Viller; qu'en la soustrayant donc avant son passage par le compteur, et ce, à l'aide de moyens frauduleux, le prévenu s'est rendu, par suite, coupable du délit de vol retenu à sa charge par les premiers juges dont il échet de confirmer la décision, tant sur la déclaration de culpabilité que sur la peine prononcée et les dommages-intérêts alloués à la partie civile;

Par ces motifs,

Reçoit en la forme l'appel de Viller envers le jugement du Tribunal de Nancy du 7 mai 1904 ;

Au fond :

Déclare ledit appel mal fondé, le rejette;

Confirme, en conséquence, le jugement attaqué dans toutes ses dispositions:

Condamne Fabius Henrion, partie civile, à tous les dépenș de première instance et d'appel, sauf son recours contre. Viller, qui devra les supporter en définitive;

Fixe au minimum contre Viller la durée de la contrainte par corps, s'il y a lieu de l'exercer.

\section{TRIBUINAI CIVII DE PAU}

Audience du 10 décembre 1904.

Présidence de M. Dubois de Lhermont, président.

RESPONSABILITÉ DES ACCIDENTS PAR I'ÉLECTRICITÉ.

I. Tout propriétaire d'un bâtiment est responsable des défauts qui $y$ sont inhérents. Or, il faut assimiler aux batiments les choses qui en dépendent par incorporation, tels que les mécanismes et les appareils.

II. Le danger d'une installation électrique étant connu, toutes les précautions possibles doivent être prises pour $y$ parer.

III. Lorsqu'un enfant est victime d'un accident mortel, les Tribunaux doivent, pour fixer la réparation due aux parents, apprécier, avec le dommage moral, le dommage que leur cause la perte du salaire de l'enfant et l'ássistance qu'ils devaient én espérer pour l'avenir.

\section{(S... contre Société Electrieue.)}

Ainsi jugé par la décision suivante:

Le Tribunal,

$\mathrm{Vu}$ l'assignation du 30 juin 3904 .

Attendu qu'il est constant que le 28 avril igo4, le jeune $S_{4}$ âgé de moins de I 4 ans, apprenti jockey chez X..., a été viçtim d'un accident mortel; qu'il a été foudroyé en touchant lêt câble conducteur d'un courant électrique à haute tension;

Attendu qu'il a été expliqué que la Société électrique dés Pyrénées, pour distribuer la lumière, a établi dans divèl endroits des appareils dits transformateurs, qui: reçoiven l'énergie électrique venant de l'usine centrale, la transforment et la conduisent dans les différents immeubles d'un même quar: tier au moyen des fils s'amorçant sur le câble du transforma teur; que l'éclairage électrique a été-installé depuis plusieurs années dans la propriété de X... par les soins de la Sóciéte électrique ; que le transformateur. a d'abord été placé aưdestuts des écuries, les-fils à havte-tensión passant au-dessus-des 
immeubles habités; mais que dès le mois d'avril I904, la Société a installé dans un coin du parc de X... une guérite spéciale pour le transformateur, avec précision fournie par la Société défenderesse qu'elle a choisi, - pour amener le courant Socte tension dans cette guérite, un câble particulier qui aurait présenté des garanties absolues d'isoliement, même au milieu de l'humidité.

Attendu que S... soutient, à juste titre, que la Société électrique est responsable du déplorable accident dont il s'agit; qu'il résulte, en effet, des documents produits que ladite société a disposé dans le parc de X ... et à la sortie de la petite cabane dans laquelle elle avait placé le transformateur, un câble à tension normale d'environ 3000 volts, à I mètre 22 cent. audessus du sol; que ce câble qui présentait un extraordinaire danger était accessible au personnel de X... et mieux, à toute personne;

Attendu qu'il est bien plaidé, au nom de la Société, que le demandeur devrait avant tout prouver la faute, la négligence et l'imprudence de cette Société; qu'il est bien spécifié que ladite Société a fait tout le possible pour éviter l'accident, puisque l'isolant du câble était, d'après le constructeur, suffisant pour que l'humidité ne soit pas à redouter et que, d'autre part, deux plaquettes avaient été apposées sur les poteaux adducteurs des fils avec l'inscription suivante : "Il y a danger de mort de toucher ces fils $\gg$;

Mais attendu que ces explications restent insuffisantes;

Que la prudence la plus élémentaire exigeait qu'un câble aussi dangereux fut placé hors de toute portêe; que la Société électrique semble même l'avoir reconnu puisqu'il a été déclaré pendant le cours des débats et non dénié par elle, qu'une barrière protectrice avait été placée depuis le jour de l'accident et que le câble avait été enfermé dans une gaîne dépassant la hauteur d'un homme, empêchant ainsi toute atteinte sans forcer la barrière ou grimper sur le poteau;

Attendu que si X... a autorisé la Société à placer une cabane ou guérite dans son parc, il n'est pas douteux pour le Tribunal que la surveillance de cette cabane et du câble qui en était l'accessoire incombait à la Société ; qu'en admettant même que le câble ait pu perdre sa puissance d'isolement par suite des pluies ou des variations atmosphériques, il appartenait à cette Société de prendre les mesures nécessaires pour remédier à cet état de choses; que le danger était connu ou tout au moins prévu, toutes les précautions possibles devaient être prises pour y parer:

Attendu, d'ailleurs, qu'aux termes de l'article $\mathrm{I} 386$, tout propriétaire d'un bâtiment est responsáble des défauts qui y sont inhérents; qu'il faut précisément assimiler aux bâtiments les choses qui en dépendent par incorporation, tels que les mécanismes et les appareils qui en font partie intégrante,

Vu les articles 1382 , r 383 et 1384 du.C. civ.;

Et, attendu qu'il est de principe, que lorsqu'un enfant á été victime d'un accident mortel, les Tribunaux doivent, pour fixer ta réparation due aux parents, apprécier avec le dommage moral, le dommage que leur cause la perte des salaires que gagnait l'enfant et l'assistance qu'ils devaient en espérer pour lavenir ;

Qué, dáns l'espèce, la situation du demandeur serait non seu. fentment très pénible, mais très précaire; que le père pouvait fégitimement compter sur son en fant qui, logé et nourri, gagnait déjà 20 francs par mois et dont les salaires pouvaient être augmentés à brève échéance;

Attendu, cependant, que poùr l'évaluation des dommagesintérêts, le Tribunal doit remarquer que la malheureuse vicfime a commis elle-même une imprudence manifeste; que sächant lire et écrire, cet enfant pouvait remarquer les deux plaqueśdont il a été parlé ci-dessus, et qu'en effet ce n'est pas par simple inattention ni mégarde qu'il a trôlé le câble électrique ;

Vú l'article i 30 du Code de procédure civile ;

Par ces motifs,

Condamne la Société électrique à payer à $\mathrm{S}$... la somme de 2.000 francs, à titre de dommages-intérêts pour les causes dont Il s'agit, avec les interêts, à compter du jour de la demande;

La condamne aux dépens.

\section{Nouveau type de Locomotive électrique}

Le 12 novembre I904, on a expérimenté une locomotive électrique d'un type tout nouveau, construite par la General Electric Company et 'par l'American Locomotive Company pour le service des trains râpidés de la ligne $\mathrm{New}$ York-Croton et du trónçon de Harlem que la Nèw York Central and Hudson River Räilroad Companýy á récemment équipés élèctriquement.

La disposition des moteurs électriques dè cette voitùre diffère totalement de celle qui est généralement adoptée dans les types ordinaires. Aussi nous a-t-il paru intéressant d'en donner ici une description d'après l'Ingegneria Ferroviaria et le Railway Age.

Cette locomotive est supportée par six paires de roues dont quatre sont motrices. Le poids de la voiture se transmet au châssis et aux essieux au moyen de balanciers en acier forgé, ainsi que cela se fait d'habitude pour répartir la charge d'une façon rationnelle.

La caractéristique principale de cette locomotive est la suivante : les induits des moteurs sont bobinés directement sur les essieux moteurs et le circuit magnétique inducteur est constitué par le chassis de la voiture. On réalise ainsi la disposition la plus simple qu'il paraisse possible d'imaginer. La figure ci-jointe permet de se rendre facilement compte du système.

Les tôles des induits sont directement calées sur les essieux moteurs, et l'enroulement induit est bobiné dans les rainures ménagées dans ces tôles. Les faces polaires sont légèrement concaves dans la partie centrale et: l'entrefer varie de trois-quarts de pouce à un pouce (19 à $25 \mathrm{mms}$ ). Le plus grand déplacement vertical que puisse prendre l'induit par rapport aux pièces polaires est de trois pouces (75 mms); ce mouvement vertical est limité par des pièces métalliques solidaires de la suspension. Les porte-balais sont fixés sur le châssis par des isolateurs montés sur des étriers, de manière que leurs positions respectives soient indépendantes des oscillations du véhicule, et leurs axes sont réglables pour pouvoir compenser l'usure des balais produite par le frottement de ceux-ci sur le collecteur.

Le changement d'un induit grillé ne paraît pas plus difficile que dans les autres itypes de commande électrique; cela revient à changer un axe dans úne locomotive ordinaire.

Le courant nécessaire est normalement capté sur un troisième rail au moyen de huit frotteurs, dont quatre de chaque côté de la locomotive. Cependant, dains les stations importantes, où les croisements sont très nombreux, la prise de courant se fait par le haut, au moyen de frotteurs spéciaux que l'on peut voir représentés sur la toiture de la locomotive. Les appareils qui supportent les frotteurs aériens sont amovibles, on peut les monter ou les abaisser à volonté, de l'intérieur de la locomotive, au moyen d'air comprimé pris sur la commande des freins..

La cabine de commande de la locomotive se trouve au centre de la voiture Là sont-les contrôleurs; les rhéostats, les appareils de mesure, voltmètres, ampèremètres, les coupe-circuits, les mécanismes de commande des freins', des sablières, des sifflets, etc. Tous cess appareils, qui sónt en double pour la marche avant et pour la marche arrière, sont du type Sprague-General Electric.

On peut réaliser trois montages différents pour la commande des moteurs électriques: Io Les quatre moteurs sont en série (petite vitesse); $2^{\circ}$ Les moteurs sont en série 